\title{
In vivo imaging of inhibitory, GABAergic neurons by MRI
}

\author{
Markus Aswendt a , Eliana Gianolio ${ }^{\text {b }}$, Giorgio Pariani ${ }^{\mathrm{b}}$, Roberta Napolitano ${ }^{\mathrm{b}}$, Franco Fedeli ${ }^{\mathrm{b}}$, \\ Uwe Himmelreich ${ }^{\mathrm{c}}$, Silvio Aime ${ }^{\mathrm{b}}$, Mathias Hoehn ${ }^{\mathrm{a}, *}$ \\ a In-vivo-NMR Laboratory, Max Planck Institute for Neurological Research, Cologne, Germany \\ b Department of Chemistry IFM and Molecular Imaging Center, University of Torino, Torino, Italy \\ c Biomedical Nuclear Magnetic Resonance Unit, Katolieke Universiteit Leuven, Leuven, Belgium
}

\section{A R T I C L E I N F O}

\section{Article history:}

Accepted 12 May 2012

Available online 18 May 2012

\section{Keywords:}

Responsive MRI contrast agent

Stem cell differentiation protocol for

GABAergic neurons

Glutamic acid decarboxylase

In vivo MRI of GABAergic neurons

\begin{abstract}
A B S T R A C T
The unambiguous detection of specific neuronal subtypes is up to now only possible with invasive techniques or optical imaging after genetic modification. High field magnetic resonance imaging (MRI) has the ability to visualize the brain structure and anatomy noninvasively, with high resolution - but missing the cell specific and functional information. Here we present a new tool for neuroimaging with MRI, enabling the selective detection of GABAergic neurons under in vivo conditions. The specific imaging contrast is achieved by a novel paramagnetic contrast agent, which responds to the activity of the enzyme glutamic acid decarboxylase - expressed solely by inhibitory neurons. The relaxivity of the complex is increased upon decarboxylation of two glutamic acid moieties, thus allowing increased water access to the inner and outer coordination spheres of the paramagnetic ion. The mechanism and specificity of activation were proven with tissue lysates and further applied to a differentiation protocol for murine embryonic stem cells. The relaxation enhancement was studied quantitatively and revealed decreased longitudinal relaxation times in the inhibitory neuron samples compared to the naïve stem cells in vitro and in vivo. Furthermore, this approach offers not only the discrimination of inhibitory, GABAergic neurons in the brain but also may expand the usefulness of MRI for functional imaging on a cellular level.
\end{abstract}

(c) 2012 Elsevier Inc. All rights reserved.

\section{Introduction}

The implantation of stem cells into the acutely or chronically damaged brain is expected to enhance the endogenous repair mechanisms through secretion of specific growth factors and to replace tissue by differentiation into the appropriate cell type. Magnetic resonance imaging (MRI) has become the method of choice for tracking stem cells because of the high spatial resolution and the possibility for repeated, noninvasive measurements (Hoehn and Himmelreich, 2006; Modo et al., 2005). A common method for tracking cells longitudinally is the labeling prior to implantation with paramagnetic or superparamagnetic contrast agents (Hoehn et al., 2008). Although conventional contrast agents highlight structural details reliably, namely the cell localization, they disguise major biological issues, e.g. cell viability and cellular processes like proliferation and differentiation. The ability to measure these biological readouts is the prerequisite for new molecular imaging approaches targeting the successful monitoring of cell implantations and their functional fate.

One possibility to overcome the common limitations is the use of contrast agents, which change their magnetic properties in response to dynamic physiological and metabolic properties, generally referred

\footnotetext{
* Corresponding author at: In-vivo-NMR Laboratory, Max Planck Institute for Neurological Research, Gleuelerstrasse 50, D-50931 Köln, Germany. Fax: + 492214726337. E-mail address: mathias@nf.mpg.de (M. Hoehn).
}

to as responsive contrast agents. Many of these contrast agents are lanthanide chelates that require direct interaction of water with the lanthanide ion. If this interaction is prevented by a functional group that can be removed by the targeted enzyme, the expression of this enzyme can be visualized by the responsive contrast agent (Himmelreich et al., 2006; Louie et al., 2000).

We focus in this report on a new MRI contrast agent to visualize specifically inhibitory neurons, which use gamma-Aminobutyric acid (GABA) as a neurotransmitter. These inhibitory neurons are very widespreadly distributed throughout the brain and comprise approximately $30-40 \%$ of all neurons (Paul, 1995). Imaging of GABAergic neurons would be important because of their pivotal role in neuronal network development, function and plasticity (Di Cristo, 2007). A decreased GABA neurotransmission is associated with neurological disorders, e.g. stroke, Huntington's disease (HD), epilepsy, schizophrenia, and several mood disorders (Benes et al., 2007; Brambilla et al., 2003; Malizia et al., 1998). Cell implantations, which aim to restore brain function by cell replacement and induction of endogenous repair mechanisms, are promising for the treatment of stroke as well as Huntington's disease. Both are associated with the loss of inhibitory neurons - in the case of HD restricted to the cortex and striatum (Deng et al., 2004; Reiner et al., 1988) and in the case of stroke restricted to the hippocampus and the striatum (Saji et al., 1994; Schwartz-Bloom and Sah, 2001). We present here a possibility to discriminate noninvasively between undifferentiated cells and GABAergic neurons after differentiation. For this purpose, we used a novel contrast 
agent, which is responsive to the enzyme glutamic acid decarboxylase (GAD), an enzyme expressed by all GABAergic neurons and responsible for converting glutamate into GABA. In this study, GAD cleaves selectively a functional group responsible for steric hindrance of water access to the coordination sphere of the paramagnetic Gd(III) ion, leading to an increase in $\mathrm{T}_{1}$ relaxivity. The targeting of a neuron-specific enzyme provides important advantages for obtaining high image contrast, including increased sensitivity and specificity. First we characterized the selective relaxivity change in correlation to the GAD content in tissue lysates. Secondly, the compound was tested on a cell culture model of undifferentiated and differentiated embryonic stem (ES) cells. Finally, implanted GABAergic neurons were discriminated due to the selective activation of the incorporated contrast agent.

\section{Materials and methods}

\section{Responsive contrast agent}

Gd-DO3A-GAD （1-[5-[[N,N'-bis-[3,10,17-triaza-4,11,18-trioxo-21carboxyl-21-aminoeinecosane]]- $N$-methylaminocarbonyl]pentyl]-1,4,7, 10-tetraazacyclododecane-4,7,10-triacetate (3-) gadolinate) (Fig. 1A) is a novel contrast agent responsive to the enzyme GAD, which catalyzes the conversion of glutamate into the major inhibitory neuron transmitter GABA (Fig. 1B). The paramagnetic agent consists of Gd-DO3A as the $\mathrm{Gd}(\mathrm{III})$ chelate backbone. In its inactive state, long hydrocarbon side chains are anchored to one $\mathrm{N}$ atom of the $\mathrm{Gd}$ (III) chelate with glutamate moieties as head groups, which can enter the Gd(III) coordination sphere, thus limiting the water access to the paramagnetic core (Fig. 1C). Upon decarboxylation of the glutamate moieties due to the GAD activity, there is an increase in the hydration sphere of the $\mathrm{Gd}(\mathrm{III})$ ion, leading to an increased $\mathrm{T}_{1}$ relaxivity. The contrast agent synthesis and chemical characterization are reported in detail elsewhere (Napolitano et al., in press).

\section{Tissue lysates and immunoblotting}

Adult Wistar rat tissues (liver, cortex and cerebellum) were mechanically homogenized, and lysed with cell lysis reagent M-PER (Thermo Fisher Scientific, Schwerte, Germany). Protein content was determined with the BCA protein assay (Thermo Fisher Scientific). Total lysate protein (30 $\mu$ g per lane) was subjected to SDS-polyacrylamide gel electrophoresis (Laemmli, 1970) and transferred to a nitrocellulose membrane (Thermo Fisher Scientific) using an electrophoresis and blotting module (Bio-Rad, Munich, Germany). Membranes were blocked with 5\% non-fat dry milk in Tris-buffered saline with Tween 20 (TBST) buffer for $1 \mathrm{~h}$. Blots were then immunolabeled for $2 \mathrm{~h}$ at room temperature (RT) against either GAD65/67 (rabbit, polyclonal, 1:750; Millipore, Darmstadt, Germany) or alpha-tubulin (mouse, monoclonal, 1:5000; Calbiochem, Darmstadt, Germany). This was followed by incubation with either anti-rabbit or anti-mouse secondary antibodies coupled to horseradish peroxidase at dilutions of 1:5000 (GE Healthcare, Freiburg, Germany). Immunolabeling was detected on an X-ray film using a chemoluminescent mixture (consisting of $90 \mathrm{mM}$ p-coumaric acid, $250 \mathrm{mM}$ luminol and $13.5 \mathrm{mM} \mathrm{H} \mathrm{O}_{2}$ in Tris- $\mathrm{HCl} \mathrm{pH} \mathrm{8.5;} \mathrm{Sigma-Aldrich,}$ Schnelldorf, Germany).

For evaluation of contrast agent activation the tissue lysates from brain (cerebellum and cortex mixed) containing equal amounts of total protein $\left(3.0-3.5 \mathrm{mg}\right.$ ) were incubated at $37^{\circ} \mathrm{C}$ for $24 \mathrm{~h}$ with $1 \mathrm{mM} \mathrm{Gd}-$ DO3A-GAD and $0.05 \mathrm{mM}$ of the cofactor pyridoxal-5'-phosphate (PLP) (Sigma-Aldrich) in PBS buffered solution. In one of the experiments, the carbonyl-trapping agent aminooxyacetic acid (AOAA), which binds to the cofactor PLP (Roberts and Simonsen, 1963), was added to the brain lysate incubation mix at $250 \mu \mathrm{M}$.

\section{GABAergic cell system}

CGR8 murine embryonic stem cells (129/Ola derived) were a generous gift from Prof. A. Sachinidis (Institute for Neurophysiology, University at Cologne, Germany). They were routinely maintained in undifferentiated state in GMEM (Glasgow Minimum Essential Medium) supplemented with 10\% FCS (ES cell tested) (both from Gibco, Darmstadt, Germany), 2 mMl-glutamine (PAA, Pasching, Austria), 100 units/ml LIF (Millipore) and $50 \mu \mathrm{M}$ beta-mercaptoethanol (Gibco) on cell culture flasks coated with $0.2 \%$ gelatine (Sigma-Aldrich). Embryoid body (EB) formation was induced by plating $23,000 \mathrm{cells} / \mathrm{cm}^{2}$ on bacteriological grade petri dishes (Sarstedt, Nürnbrecht, Germany) in GMEM supplemented with $10 \%$ FCS (ES cell tested) and 2 mML-glutamine (PAA) for 3 days. Neural precursor cells (NPCs) were selected over 4 days by culturing EBs on bacteriological grade cell culture dishes in ITSFn
A

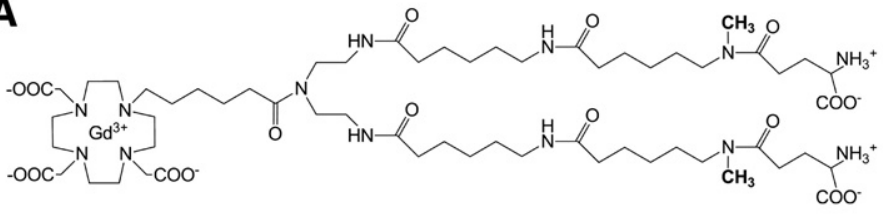

B

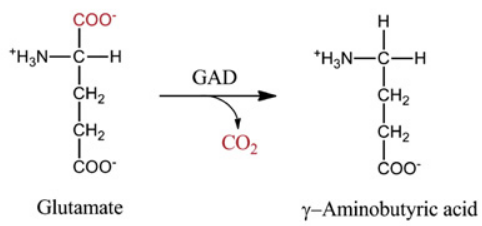

C

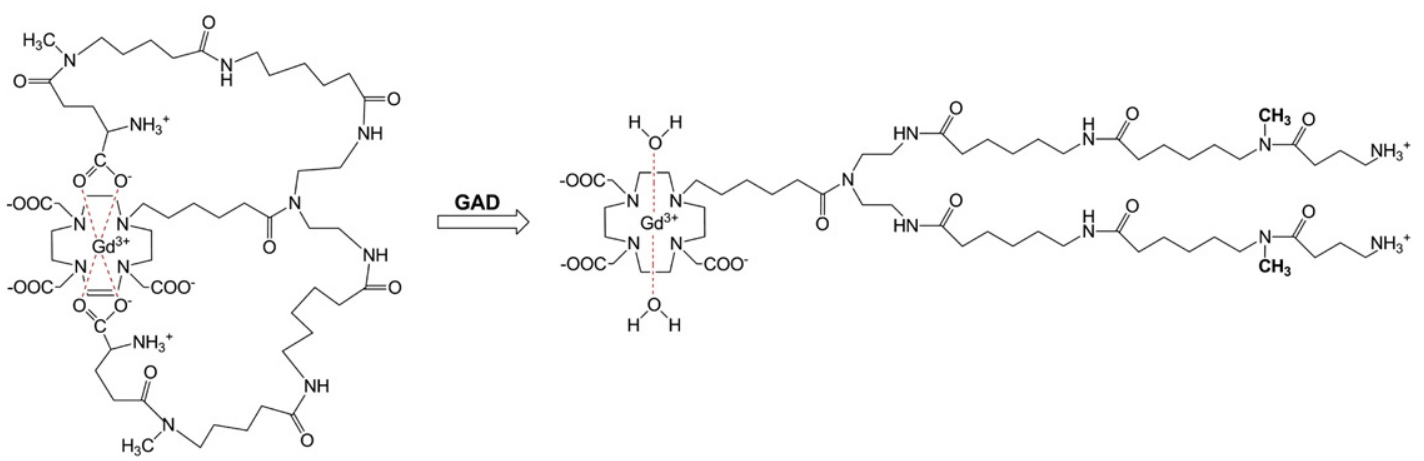

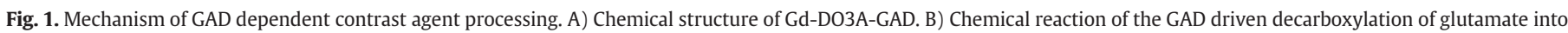
GABA. C) The glutamate moieties coupled to the Gd(III) core of the complex are cleaved by GAD leading to free access of water and higher relaxivity of the contrast agent. 
medium, which consists of DMEM/F12 (Dulbecco's Modified Eagle Medium) (Gibco), $1 \times$ ITS (Sigma-Aldrich), 2 mMl-glutamine (PAA) and $1 \mathrm{ng} /$ $\mathrm{ml}$ fibronectin (Sigma-Aldrich). NPC spheres were mechanically detached, centrifuged at $163 \times \mathrm{g}$ and plated on polyornithine/laminin covered dishes in neuronal differentiation medium for at least 3 days (polyornithine/laminin coating consists of $18 \mathrm{ng} / \mathrm{ml}$ poly-L-ornithine and $5 \mu \mathrm{g} / \mathrm{ml} \mathrm{laminin}$ (Sigma-Aldrich)). The neuronal differentiation medium was KnockOut DMEM (Gibco), 10\% KnockOut serum replacement (Gibco), $1 \times$ NEAA (PAA), 2 mM L-glutamine (PAA). All cells were cultured under humidified $5 \% \mathrm{CO}_{2}$ and $95 \%$ air. For immunocytochemical analysis, cells were plated on polyornithine/laminin covered glass slides.

\section{Gene expression analysis}

For quantitative real time polymerase chain reaction (qPCR) experiments, total RNA was isolated using the Nucleo Spin RNA II Kit (Macherey-Nagel, Düren, Germany) and $5 \mu \mathrm{g}$ of purified total RNA were used for single strand cDNA synthesis using the Omniscript RT Kit (Qiagen, Hilden, Germany). For primer design QuantPrime was used (Arvidsson et al., 2008) based on the NCBI reference sequences for mouse mRNA (National Center for Biotechnology Information, Bethesda, USA). The sequence of the primers were as follows, beta IIItubulin: 5'-ATG AGG CCT CCT CTC ACA AGT ATG-3' and 5'-T CC AGG TTC CAA GTC CAC CAG-3'; glutamic acid decarboxylase 1 (GAD1): 5'CAG CCA GAC AAG CAG TAT GAC G-3' and 5'-TTG CTT TCC ACA TCA GCC AGA AC-3'; glutamic acid decarboxylase 2 (GAD2): 5'-ACC ACA ATG GTC AGC TAC CAA CC-3' and 5'-TTT GAG ATG ACC ATG CGG AAG AAG-3'; glyceraldehyde-3-phosphate dehydrogenase (GAPDH): 5'CAT GGC CTT CCG TGT TCC TA-3' and 5'-CG GCA CGT CAG ATC CA-3'; Nestin: 5'-AGT GGC TAC ATA CAG GAC TCT GC-3' and 5'-TTC TTC CAG GTG TCT GCA AGC G-3'; Oct4: 5'-TGT TTC TGA AGT GCC CGA AGC C-3' and 5'-CCA AGC TGA TTG GCG ATG TGA G-3'. GAD1 and GAD2 correspond to the gene coding for GAD67 and GAD65 respectively. GAPDH was chosen as a robust housekeeping gene, because of the reported stable expression in mouse embryonic stem cells and neural differentiation protocols (Willems et al., 2006). qPCR experiments were conducted with the SensiMix SYBR No-ROX kit (Bioline, Luckenwalde, Germany) and the TOptical thermocycler (Biometra, Göttingen, Germany) according to the protocol of the manufacturer. Relative quantification based on the ÄÄCT method was achieved acquiring the PCR efficiencies for each gene of interest with a cDNA mixture and the cycle threshold (CT) values for each specific gene and CDNA in duplicate. The data was analyzed using the Biometra RT PCR software and the Relative Expression Software Tool V2.0.13 (Pfaffl et al., 2002).

\section{Immunostainings}

For immunocytochemistry (ICC) cells were fixed in 4\% paraformaldehyde (PFA) in PBS for 15 min at RT, and washed three times in PBS. Cells were permeabilized for 10 min with $0.03 \%$ Triton X-100/TBS, and unspecific antibody binding was blocked with $5 \%$ BSA/TBS (PAA) for $1 \mathrm{~h}$. Primary antibodies were applied for $2 \mathrm{~h}$ in $0.8 \%$ bovine serum albumin (BSA)/TBS and secondary antibodies for $2 \mathrm{~h}$ in TBS. The following antibodies were used: beta III-tubulin (mouse, monoclonal, 1:300, Sigma-Aldrich), GABA (rabbit, polyclonal, 1:1000, Sigma-Aldrich), GAD65/67 (rabbit, polyclonal, 1:200, Millipore), Dylight 488 (goat anti mouse, 1:1000, Thermo Fisher Scientific) and DyLight 549 (goat anti rabbit, 1:1000, Thermo Fisher Scientific). Nuclei were counterstained for $10 \mathrm{~min}$ by Hoechst $33342(1 \mu \mathrm{g} / \mathrm{ml}$, Sigma-Aldrich). Cells were mounted within aquamount (Thermo Fisher Scientific) and visualized under a $40 \times$ objective on a Leica TCS SP2 microscope equipped with a supplementary CCD camera (Leica, Microsystems, Wetzlar, Germany). Quantification of cell differentiation was achieved by counting GABA, $\mathrm{GAD}$, beta III-tubulin positive cells and total cell number on 5 randomly chosen fields of view.
For histology, mice were perfused transcardially under isoflurane anesthesia, first with saline followed by 4\% PFA. Brains were removed and post-fixed overnight in PFA, followed by $20 \%$ sucrose solution until they sunk. Thirty-micrometer-thick sections were cut in the coronal plane using a cryostat (Leica) and stored at $-20^{\circ} \mathrm{C}$. The hematoxylin and eosin (HE) stain was applied to selected sections. Briefly, slides were washed in several ethanol solutions (100\%, 90\% and 70\%), incubated with hemalaun (Merck, Darmstadt, Germany) followed by washing in distilled water and tab water, incubation in eosin (Merck), washing in distilled water followed by increasing EtOH solutions. Slides were mounted with Entellan (Merck) after washing in Roti-Histol (Carl Roth, Karlsruhe, Germany).

\section{Cell labeling}

Several transfection methods commonly used in molecular biology to introduce foreign DNA in cells were compared with regard to the uptake of the commercially available paramagnetic contrast agent Dotarem ${ }^{\circledR}$ (Guerbet, Roissy CdG Cedex, France) and the cell viability/survival rate. The tested methods are 1) Pinocytosis (Pino): during direct incubation of the contrast agent in the cell medium, spontaneous uptake takes place by random pinocytosis/ endocytosis (Terreno et al., 2006), 2) calcium phosphate (CaP): a coprecipitate of $\mathrm{CaP}$ and the contrast agent enter the cell by endocytosis (Graham and van der Eb, 1973), 3) lipofection (Lipo): the contrast agent is coated by a lipid layer which induces cell uptake (Rudelius et al., 2003), and 4) electroporation (EP), which induces the reversible formation of small pores in the cell membrane upon an electrical discharge (Neumann et al., 1982). Cells were prepared as follows: undifferentiated CGR8 cells were detached with $0.05 \%$ trypsin/0.02\% EDTA (PAA), centrifuged at $250 \mathrm{xg}$ for $3 \mathrm{~min}$ and counted. Differentiated CGR8 cells were detached and dissociated with accutase (PAA) by careful titration, centrifuged at $250 \mathrm{xg}$ for $3 \mathrm{~min}$ and counted.

Pinocytosis was achieved by incubating the cells for $2 \mathrm{~h}$ in the presence of $50 \mathrm{mM}$ Dotarem ${ }^{\circledR}$. For lipofection a custom-made transfection agent similar to lipofectamine (Invitrogen, Darmstadt, Germany) (Kruttwig, 2009) was used in a lipo:contrast agent ratio of $1: 2$, preincubated for $30 \mathrm{~min}$, followed by $2 \mathrm{~h}$ incubation with the cells in complete medium. The CaP transfection mixture was prepared as described by (Chen and Okayama, 1987). Briefly, $2 \times$ BES-buffered saline (BBS), $0.25 \mathrm{M} \mathrm{CaCl}_{2}$ and $50 \mathrm{mM} \mathrm{Gd-DOTA}$ were mixed in a ratio of 2:1 and treated like the lipofection samples. For electroporation 50 mM Gd-DOTA were mixed with ice-cold Hanks Balanced Salt Solution (HBSS) (Invitrogen) and directly electroporated using a Genepulser (Bio-Rad, Vienna, Austria) with a $100 \mathrm{~V}$ square pulse for $20 \mathrm{~s}$. After EP, cells were placed on ice for $5 \mathrm{~min}$. Independent of the labeling method, cells were finally washed twice with PBS to remove excessive contrast agent and counted by the trypan blue exclusion assay to determine viability after labeling (viability $=100 \% \times$ viable cells/total cell number). Furthermore, the survival rate was used to determine the actual cell death due to the treatment (survival rate $=100 \% \times$ viable cells $s_{\text {with }}$ treatment $/$ viable cells $_{\mathrm{w} / \mathrm{o}}$ treatment $)$. The electroporation protocol was applied to undifferentiated and differentiated cells with $10 \mathrm{mM} \mathrm{Gd-DO3A-GAD}$. The cells were subsequently plated for $6 \mathrm{~h}$ and collected by centrifugation at $250 \times \mathrm{g}$ for $3 \mathrm{~min}$. Labeled cells were washed and counted as described above. Cell pellets were either fixed with PFA for $15 \mathrm{~min}$ at RT or directly used for cell implantations adjusted to $1.875 \times 10^{5}$ cells/ $\mu \mathrm{l}$ in HBSS.

\section{Phantoms}

Phantoms for MRI experiments at $4.7 \mathrm{~T}$ consisted of plastic PCR tubes containing a mixture of tissue lysates or cells and $0.5 \%$ low melting agarose (Sigma-Aldrich) covered and embedded in 1\% agarose (SigmaAldrich). Measurements at 1.0 and 7.0 T were conducted on cell pellets 
in glass capillaries embedded in a $10 \mathrm{~mm}$ glass tube filled with $1 \%$ agarose. Agarose phantoms were cooled down to room temperature for MR experiments and stored at $4{ }^{\circ} \mathrm{C}$.

\section{Cell implantation for in vivo MRI}

All animal experiments were conducted according to the guidelines laid out in the German Animal Welfare Act, in accordance with the European Communities Council Directive 86/609/EEC, and were approved by the local authorities (Landesamt für Natur, Umwelt und Verbraucherschutz North Rhine-Westphalia, reference number 8.87-51.04.20.09.362). Nude mice (Janvier, France; $n=2$ ) were anaesthetized with Isoflurane in $\mathrm{O}_{2}: \mathrm{N}_{2} \mathrm{O}(30: 70 \%)$ and fixed in a stereotactic frame (Stoelting, Dublin, Ireland). Carprofen (Pfizer, Karlsruhe, Germany) was used for analgesia ( $4.0 \mathrm{mg} / \mathrm{kg}$ s.c.). The skull was exposed by a small incision and two holes were drilled at the following coordinates relative to bregma: $\mathrm{AP}+0.5 ; \mathrm{L} \pm 2.0 ; \mathrm{DV}-2.5$ using a stereotactical instrument (Stoelting). The homogeneous cell suspensions were kept on ice during the surgery and subsequently injected into the brain through a Hamilton syringe (23 G needle) using a micropump system with flow rates of $1500 \mathrm{nl} / \mathrm{min}$ (withdraw) and $500 \mathrm{nl} / \mathrm{min}$ (injection). In total, $7.5 \times 10^{5}$ cells were injected in a volume of $2 \mu \mathrm{l}$. Animals were kept under anaesthesia for the 0.5 hour scan post implantation and were later scanned again at $24 \mathrm{~h}$ and 7 days post implantation.

\section{Magnetic resonance imaging and relaxometry}

The experiments with tissue lysates and for the optimization of cell labeling were conducted on a Biospec $4.7 \mathrm{~T}$ (Bruker BioSpin, Ettlingen, Germany) with a Helmholtz coil for transmit (120 mm diameter) and a surface coil ( $28 \mathrm{~mm}$ diameter) for receive. $\mathrm{T}_{1}$ maps were acquired with a RAREVTR sequence including the following parameters: 12 variable $\mathrm{TR}=116.640,258.513,415.220,590.230,788.388,1016.768$, 1286.274, 1615.045, 2036.712, 2625.391, 3608.035, $7500.000 \mathrm{~ms}$, $\mathrm{TE}=12.0 \mathrm{~ms}$, field of view $(\mathrm{FOV})=30.0 \times 30.0 \mathrm{~mm}$, slice thickness $2.0 \mathrm{~mm}$, matrix $=128 \times 128$, resolution $=0.234 \times 0.234 \mathrm{~mm}$, number of averages $=2$, with a total acquisition time (TA) of $46 \mathrm{~min}$.

MR images on cell pellets were acquired on an Avance 300 spectrometer (Bruker) operating at $7.1 \mathrm{~T}$ equipped with a microimaging probe using a standard $\mathrm{T}_{1}$-weighted multi-slice multi-echo sequence $\left(\mathrm{TR}=250 \mathrm{~ms}, \mathrm{TE}=3.3 \mathrm{~ms}, \mathrm{NEX}=6, \mathrm{FOV}=0.115 \times 0.115 \mathrm{~cm}^{2}, 1\right.$ slice, slice thickness $=1 \mathrm{~mm}$ ) and on an Aspect M2 system (Aspect Imaging, Shoam, Israel) operating at $1 \mathrm{~T}$ equipped with a horizontal bore MRI magnet using a standard $\mathrm{T}_{1}$-weighted multi-slice spin-echo sequence $\left(\mathrm{TR}=250 \mathrm{~ms}, \mathrm{TE}=7.0 \mathrm{~ms}, \mathrm{NEX}=30, \mathrm{FOV}=0.17 \times 0.17 \mathrm{~cm}^{2}, 1\right.$ slice, slice thickness $=1.5 \mathrm{~mm}$ ). $\mathrm{T}_{1}$ values were measured using a saturation recovery spin-echo sequence (at $7 \mathrm{~T}$ : $\mathrm{TE}=2.6 \mathrm{~ms}, 16$ variable $\mathrm{TR}$ ranging from 40 to $6000 \mathrm{~ms}, \mathrm{NEX}=1, \mathrm{FOV}=0.115 \times 0.115 \mathrm{~cm}^{2}, 1$ slice, slice thickness $=1 \mathrm{~mm}$; at $1 \mathrm{~T}$ : $\mathrm{TE}=7.0 \mathrm{~ms}, 12$ variable TR ranging from 50 to $4000 \mathrm{~ms}, \mathrm{NEX}=1, \mathrm{FOV}=0.17 \times 0.17 \mathrm{~cm}^{2}, 1$ slice, slice thickness $=2 \mathrm{~mm}$ ). For relaxometric determination of intracellular Gd(III)-content, cell pellets were treated as described elsewhere (Arena et al., 2011).

The proton $R_{1}$ NMRD profiles on tissue lysates were measured at $25{ }^{\circ} \mathrm{C}$ on a fast field-cycling Stelar relaxometer (Stelar, Mede Pavia, Italy) over a continuum of magnetic field strengths from 0.00024 to $0.47 \mathrm{~T}$ (corresponding to $0.01-20 \mathrm{MHz}$ proton Larmor frequencies). The relaxometer operates under computer control with an absolute uncertainty in $\mathrm{R}_{1}$ of $\pm 1 \%$. Additional data points in the range of 20-70 MHz were obtained on a Stelar Spinmaster relaxometer tunable at the different frequencies. The temperature was controlled with a Stelar VTC-91 air-flow heater equipped with a copper constantan thermocouple (uncertainty $0.1^{\circ} \mathrm{C}$ ).

The in vivo MRI data was acquired on a Biospec 7.0 T (Bruker) under isoflurane (2\%) anaesthesia $\left(\mathrm{O}_{2} / \mathrm{N}_{2} \mathrm{O}, 30 / 70 \%\right)$ and lasted approximately $35 \mathrm{~min}$. For a general overview and the coregistration, a $\mathrm{T}_{2}$-weighted RARE sequence was used $(\mathrm{TR}=6500 \mathrm{~ms}$, $\mathrm{TE}=13.0 \mathrm{~ms}$, FOV $=14.0 \times 14.0 \mathrm{~mm}^{2}, 30$ slices, slice thickness $=0.5 \mathrm{~mm}, 128 \times 128$ matrix, i.e. a resolution of $\left.109 \times 109 \times 500 \mu \mathrm{m}^{3}\right)$. The $\mathrm{T}_{1}$ maps were acquired with a RAREVTR sequence (12 variable TR 189.708, 331.527, 488.167, 663.094, 861.146, 1089.384, 1358.693, 1687.171, 2108.355, $2696.085,3676.028,7500.000 \mathrm{~ms}, \mathrm{TE}=12.0 \mathrm{~ms}, \mathrm{FOV}=20.0 \times 20.0 \mathrm{~mm}^{2}$, 6 slices, slice thickness $=1.0 \mathrm{~mm}, 128 \times 128$ matrix, i.e. a resolution of $156 \times 156 \times 1000 \mu^{3}$ ).

\section{Data analysis and postprocessing}

Images from IHC and MRI were processed with ImageJ (Version 1.42q; National Institutes of Health). $\mathrm{T}_{1}$ maps were calculated using a custom-made program developed in IDL (ITT Visual Information Solutions, Boulder, USA). The MRI signal S(TR) was fitted on a voxel basis with a monoexponential function $\mathrm{S}(\mathrm{TR})=\mathrm{S}_{0} *\left(1-\exp \left(-\mathrm{TR} / \mathrm{T}_{1}\right)\right)$ with equilibrium signal $S_{0}$, longitudinal relaxation time $T_{1}$ and the scan repetition time TR. Region of interest (ROI) analysis of the quantitative maps was used to determine the $T_{1}$ values with ImageJ. In case of phantom scans, circular ROIs were drawn manually. For the in vivo measurements, two ROIs were drawn manually along the grafting canal in one animal. ROI measurements were then applied to all scans, yielding $T_{1}$ values for the phantom samples and the cell grafts, respectively. Changes in relaxation rate $\Delta \mathrm{R}_{1}$ induced by the contrast agent conversion were calculated by $\Delta R_{1}=\left(1 / T_{1 \text { (treated sample) }}\right)$ $-\left(1 / \mathrm{T}_{1 \text { (sample) }}\right)$. Further processing of the in vivo MRI data was done by converting the raw data into 32-bit NIFTI format (Neuroimaging Informatics Technology Initiative; http://nifti.nimh.nih.gov) and scaling up the voxel size by a factor of 10 . For brain extraction and affine coregistration with $12^{\circ}$ of freedom, FSL tools were used (FMRIB
A

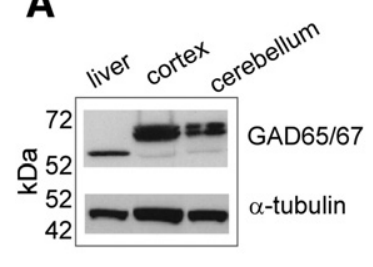

B

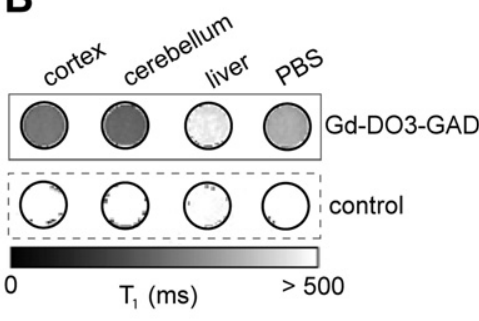

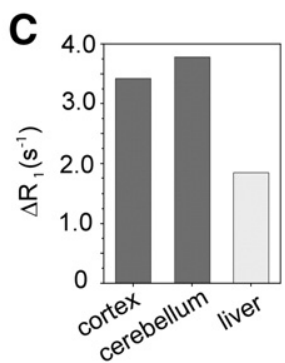

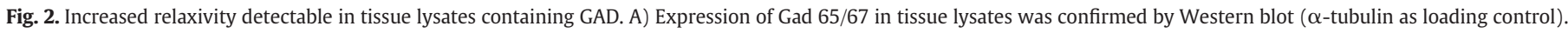

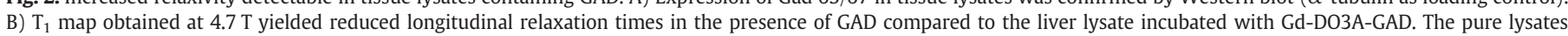
served as a control. C) Cortex and cerebellum showed approximately 2-fold increased $\Delta R_{1}$ rates in respect to the liver lysate. 


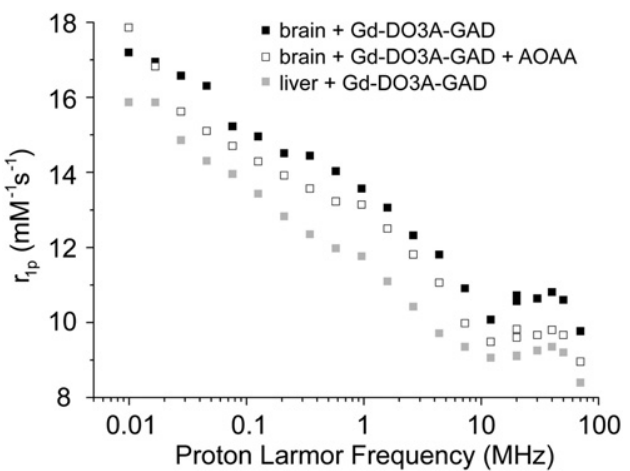

Fig. 3. Field strength behavior of the complex and specificity of GAD mediated relaxivity change. Enhancement of Gd-DO3A-GAD relaxivity was dependent on the proton Larmor frequency, proven by NMRD profiles. Cortex and cerebellum (brain) were incubated with $10 \mathrm{mM}$ Gd-DO3A-GAD and relaxivity $\mathrm{r}_{1 \mathrm{p}}$ was measured at various field strengths. The relaxivity shift due to the GAD activity was approximately $30 \%$ reduced by the addition of the GAD specific inhibitor AOAA.

Software Library; http://www.fmrib.ox.ac.uk/fsl). The data was processed according to a 4-step protocol: 1) RARE scans of all animals were registered and averaged to yield a brain template, 2) the RAREVTR scans of every time point were coregistered to the average brain, 3 ) the resulting transformation was applied to the $T_{1}$ maps accordingly and 4) for visualization, brain maps were masked manually to exclude non-brain tissue.

\section{Results}

Detection of GAD specific $T_{1}$ change in tissue extracts

Western blot was used to assess GAD65/67 protein in lysates from the rat brain (cortex and cerebellum). No GAD65/67 could be detected in the lysate of liver tissue, which served in the following as a negative control (Fig. $2 \mathrm{~A}$ ). The $\mathrm{T}_{1}$ maps of the lysates after incubation with Gd-DO3A-GAD revealed a $\mathrm{T}_{1}$ relaxation time decrease in cortex and cerebellum samples relative to liver sample (Fig. 2B). The acquired $T_{1}$ values were used to calculate $\Delta R_{1}$ of tissue samples with the contrast agent and untreated samples. The $\Delta R_{1}$ value of brain samples compared to the liver sample was approximately doubled, indicating a high relaxivity shift of the Gd-DO3A-GAD complex (Fig. 2C). The relaxivity behavior was further studied at different magnetic field strengths (Fig. 3). On the basis of the obtained NMRD profiles, the complex exhibited the highest relaxivity shift useful for MRI at $1 \mathrm{~T}$ (or $40 \mathrm{MHz}$ ). The specificity of GAD mediated contrast was confirmed by adding the GAD specific inhibitor AOAA, which decreased the relaxivity shift in brain lysates.

\section{Cell culture protocol for GABAergic neurons}

For relaxometry studies on a cellular level, a differentiation protocol for CGR8 ES cells was set up, which facilitates culturing of several million GABAergic cells in a short time. The novel three step protocol (Fig. 4A) takes 10 days and consists of 1 ) the EB formation from CGR8 cells, 2) the selection of NPCs in chemically defined medium, and 3) the final neural differentiation. This method enables reproducibly

A
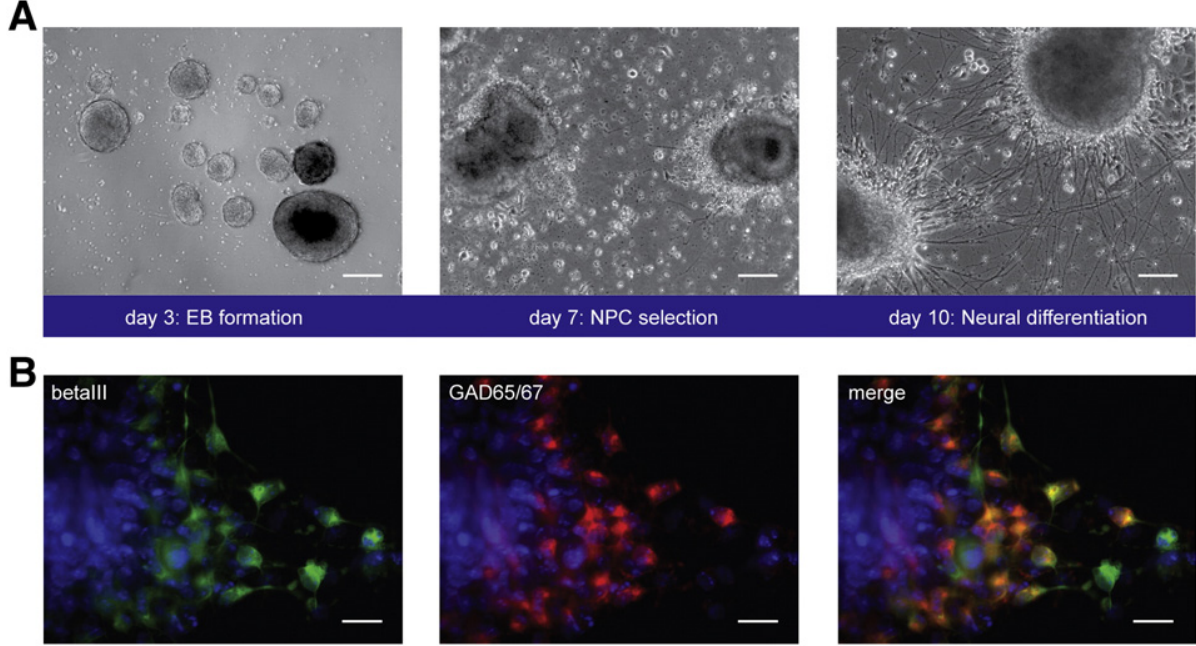

C

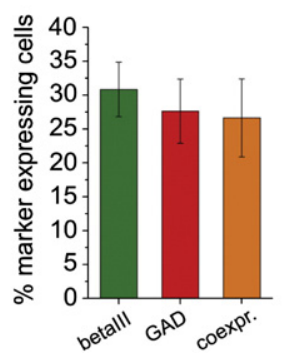

D

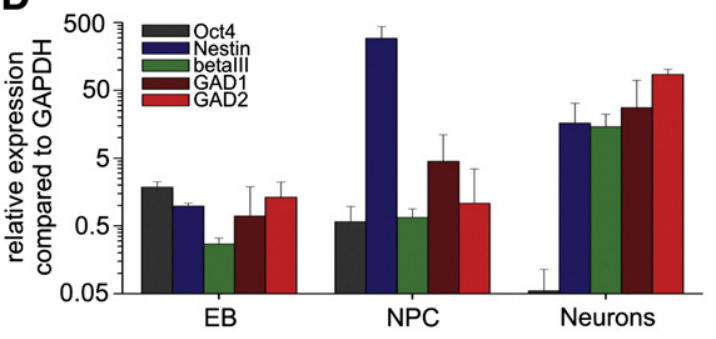

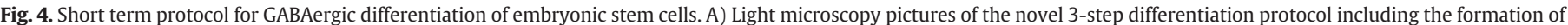

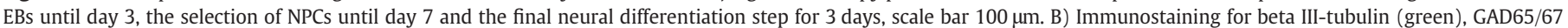

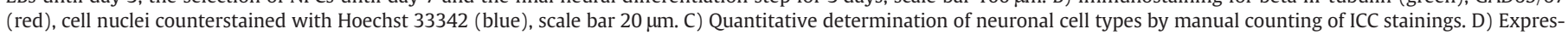

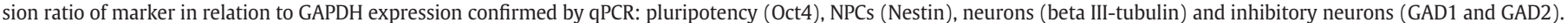


the generation of a neural culture including GABAergic neurons expressing the GAD65/67 protein, which was confirmed by ICC (Fig. 4B). Approximately $31 \%$ of all cells in the culture were beta IIItubulin positive neurons. $27 \%$ of all cells were also co-expressing GAD65/67 indicating a high degree of GABAergic neurons in the culture (Fig. 4C). The neuronal differentiation was further validated by qPCR analysis (Fig. 4D). Relative gene expression was compared to the gene expression of the housekeeping gene GAPDH in CGR8 cells. The obtained gene expression profile clearly reflects 1 ) the downregulation of the pluripotency marker Oct4, 2) the maximum Nestin expression under NPC selection conditions, and 3) the selective expression of GAD1 and GAD2 in the final step of differentiation.

\section{Successful incorporation of Gd-chelate into ES cells and GABAergic neurons}

Efficient labeling of stem cells with MRI contrast agents is hindered by the stem cell vulnerability against external influences. Particularly, the toxicity effects from incorporated contrast agents on the pluripotency status, viability and proliferation capacity need to be minimized. We compared different labeling approaches for CGR8 cells using the conventional, clinically used contrast agent Dotarem ${ }^{\circledR}$. The applied methods were 1) self-acting accumulation of contrast agent by pinocytosis/endocytosis, 2) calcium phosphate precipitation, 3) lipofection, and 4) electroporation (Fig. 5A). Pinocytosis does not affect viability or survival rate (both over $90 \%$ ). In contrast, calcium phosphate, lipofection and electroporation lead to a decreased survival rate without affecting viability of the cells, indicating a detrimental effect (least value $62 \%$ for electroporation). To determine the efficiency of contrast agent uptake, $T_{1}$ maps were acquired from MRI phantoms containing labeled cells at $4.7 \mathrm{~T}$. The calculated $\Delta \mathrm{R}_{1}$ values indicate a minimal unspecific uptake of contrast agent by pinocytosis, which could not be enhanced by calcium phosphate (Fig. 5B). Nevertheless, a stronger effect (factor 2.4) on the $\Delta R_{1}$ was observed for the samples treated by lipofection and electroporation with a more consistent labeling by electroporation. To gain high viability/survival and uptake of contrast agents together, the electroporation protocol needed optimization concerning buffer and electrical power applied, resulting in the combination of HBSS buffer and a single square wave at $100 \mathrm{~V}$ with a pulse length of $20 \mathrm{~ms}$.

The optimized labeling protocol was applied to the undifferentiated and differentiated CGR8 cells in the presence of Gd-DO3A-GAD
(Fig. 5C), in which the viability remained high (83-95\%). In contrast, cell survival decreased slightly to 53-72\%. Remaining differentiation potential was validated by culturing the labeled cells according to the described protocol, achieving a similar portion of GABAergic neurons expressing GAD for labeled and unlabeled cells (data not shown).

\section{Differentiation-specific $T_{1}$ contrast and field dependence}

The undifferentiated and differentiated CGR8 cells were labeled with Gd-DO3A-GAD using the optimized electroporation protocol, and incubated for $6 \mathrm{~h}$ to allow complete activation of the contrast agent. Fixed cells were subsequently measured in MRI phantoms at 1 and $7 \mathrm{~T}$. In Fig. 6A, an example of the obtained $\mathrm{T}_{1}$-weighted MR images at $1 \mathrm{~T}$ and $7 \mathrm{~T}$ is shown. Based on the $\mathrm{T}_{1}$ maps of 3 independent experiments obtained at $1 \mathrm{~T}$ and $7 \mathrm{~T}$, the $\Delta \mathrm{R}_{1}$ values were calculated in relation to the untreated cells (Figs. $6 \mathrm{~B}, \mathrm{C}$ ). At both field strengths, a robust increase in $\Delta R_{1}$ could be observed in the differentiated compared to the undifferentiated cells ranging from 30 to $100 \%$. The relaxation rate decreased with the higher field strength, which is consistent with the NMRD profiles of tissue lysates (Fig. 3). Further calculations were made in order to represent the relaxation rate variation of differentiated cells with respect to undifferentiated ones. For the calculated $\Delta \mathrm{R}_{1}$ differences between both samples a normalization was applied, based on the actual number of vital cells in each experiment and the $\mathrm{Gd}(\mathrm{III}) / \mathrm{mg}$ protein content (Fig. 6D). Because of the high standard deviation of these values, we conclude that the experiments revealed high fluctuations in the amount of contrast agent stably incorporated by the cells, but the relative difference in observed relaxation rates was maintained. Furthermore, the $T_{1}$ contrast at high field strength was still $\geq 100$ ms which would make in vivo applications at high fields possible.

\section{In vivo cell imaging at high field strength}

The in vivo behavior of Gd-DO3A-GAD was characterized at high field strength (7 T, Bruker Biospec) (Fig. 7A). Cell grafts of 750,000 pre-labeled undifferentiated and differentiated cells were implanted into the striatum of 2 nude mice and subsequently followed for up to 7 days by MRI. At each time point, a $\mathrm{T}_{1}$ map was obtained (Fig. 7B) and the change in relaxation rate between the ES cell graft and the differentiated graft was calculated. A loss of contrast was observed after $24 \mathrm{~h}$ and 7 days. $0.5 \mathrm{~h}$ after surgery, the two cell grafts
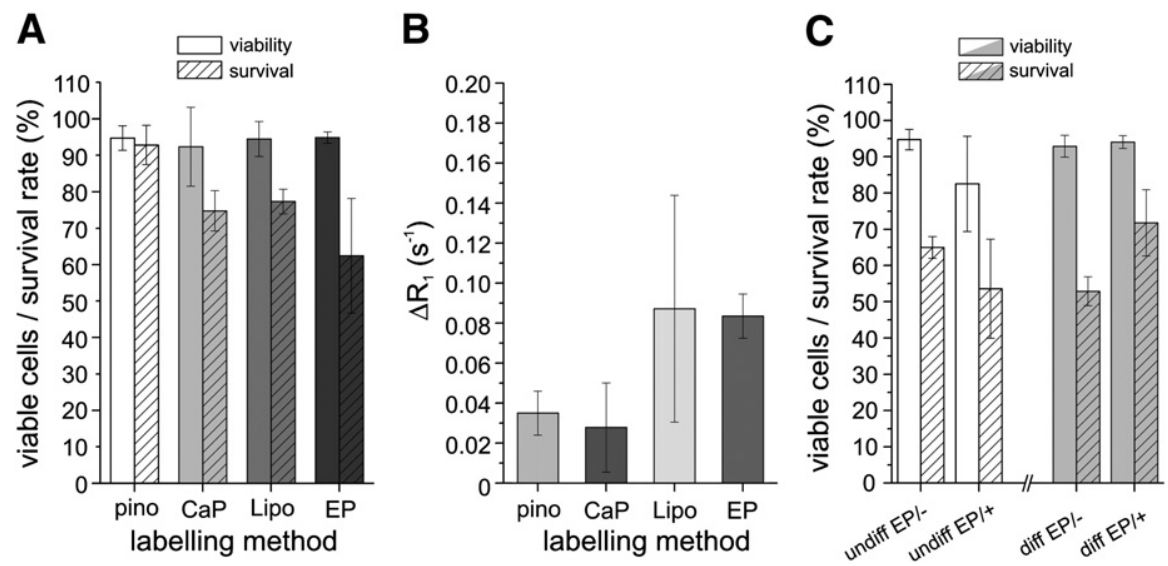

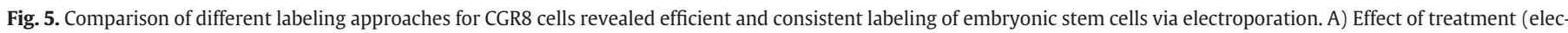

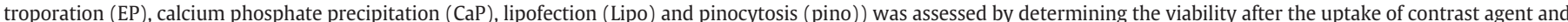

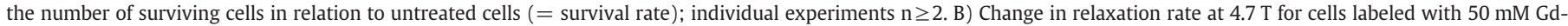

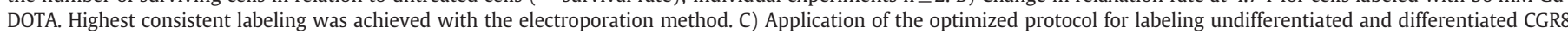

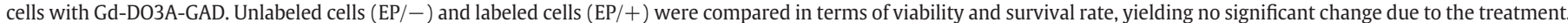


A

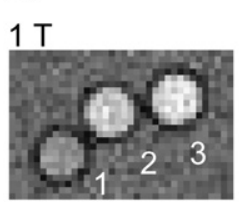

$7 \mathrm{~T}$

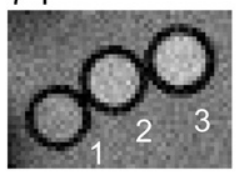

B

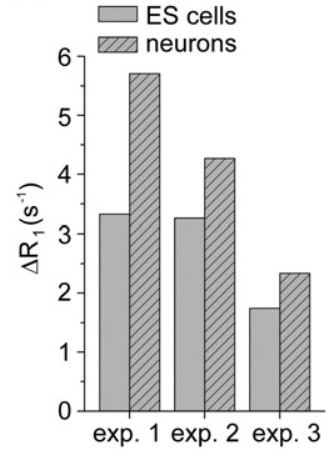

C

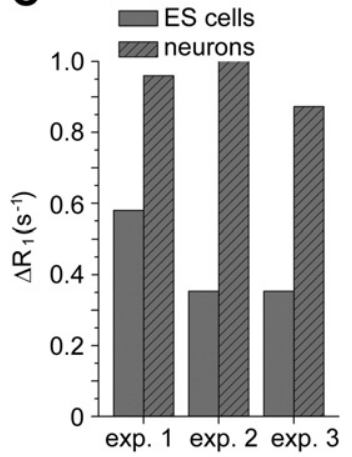

D

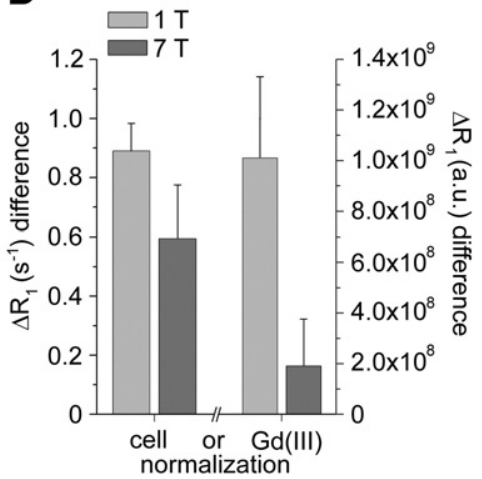

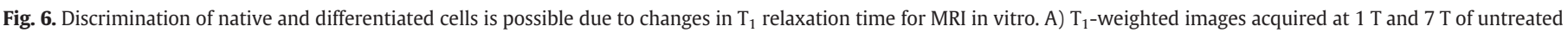

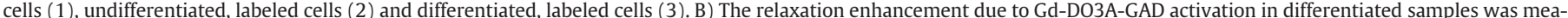

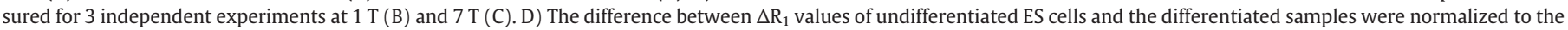

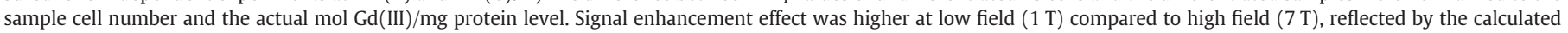
change in $\Delta R_{1}$ rates from 2 independent experiments normalized for the cell number in each sample (undifferentiated vs. differentiated cells).

could be clearly discriminated based on the decreased $\mathrm{T}_{1}$ values for differentiated cells compared to the undifferentiated cells (Fig. 7C). The histological evaluation of implant location revealed a symmetric location of both cell grafts in the striatum. The implanted cells highlight characteristically as a dense package of cells (Fig. 7D).

\section{Discussion}

To the best of our knowledge, the present investigation is the first in vivo visualization of neuron specific imaging using a novel MRI contrast agent responsive to the neuronal enzyme GAD. The GAD specific activation was demonstrated with tissue lysates and incubation in the presence of a GAD-specific inhibitor. A cellular model for GABAergic neurons was established and the contrast agent uptake route was optimized in terms of cell survival and amount of incorporated complex. Finally, the intracellular relaxivity changes due to the activation of Gd-DO3A-GAD were used to discriminate ES cells and inhibitory neurons by MRI in vitro and in vivo after implantation into the mouse brain.

\section{Selectivity of contrast agent activation}

The mechanism of Gd-DO3A-GAD activation is an advancement of the mechanism in the first reported enzyme-activated gadoliniumbased chelate by Moats et al. (1997). It is functionalized by masking groups which can be cleaved specifically by the enzyme GAD leading to an increased $\mathrm{T}_{1}$ relaxivity due to the improved access of water molecules to the $\mathrm{Gd}(\mathrm{III})$ ion. To our knowledge, the results presented here are the first neuronal application of a contrast agent responsive to an endogenous neuronal enzyme. We tested the selective relaxivity shift in tissue lysates from the brain containing GAD compared to the liver. Cortex and cerebellum were chosen because of the reported high expression of GAD (Mueller and Langemann, 1962). By incubation of the GAD-containing brain lysates in the presence of the selective inhibitor AOAA the relaxivity shift was markedly reduced. The co-factor PLP plays a crucial role for the GAD enzyme and was added to the lysates in order to enhance GAD activity under lysate conditions. The PLP seems to be essential for the GAD action on the contrast agent, because the PLP-trapping AOAA decreased the reaction efficiency. The NMRD profiles are representative for paramagnetic complexes. Deviations can be explained by the lysate composition, a crude mixture of all cellular components, which may lead to unspecific complexing of the agent to macromolecules, as was reported previously (Napolitano et al., in press).

\section{GABAergic cell model}

The relaxivity measurements on a cellular level under controlled conditions required a substantial amount of GABAergic neurons. Due to the lack of established commercial GABAergic cell lines, we used embryonic stem cells (CGR8), which can be differentiated into GABAergic neurons (Shin et al., 2011). Our novel differentiation protocol relies on the five-stage method introduced by Okabe and others (Lee et al., 2000; Okabe et al., 1996). In contrast to the established methods, we were able to shorten the differentiation time from approximately 3 weeks to 10 days and to simplify the cell culture
A

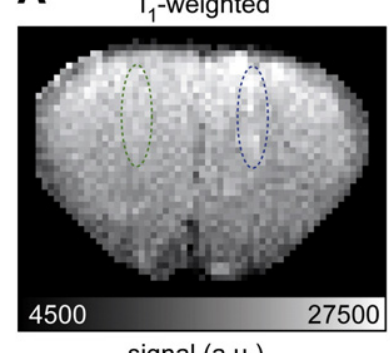

B

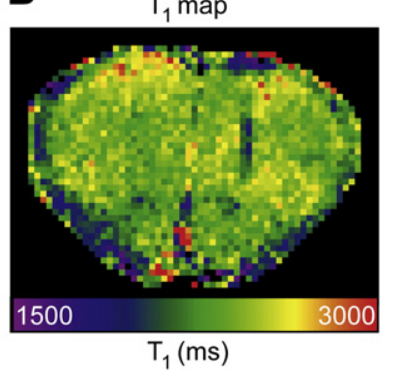

C

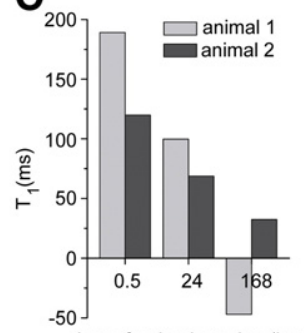

time after implantation (hours)

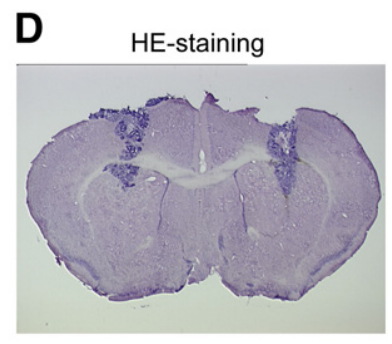

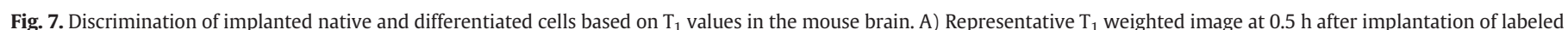

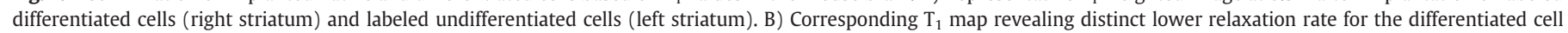

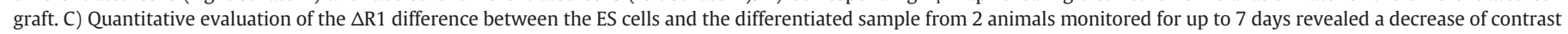
over time. D) Histological prove of the location of cell grafts after sacrificing the animal 7 days after implantation by HE-staining. 
conditions. Nevertheless, the optimized protocol yielded in total $30 \%$ neurons of which approximately $89 \%$ were GABAergic, which is in line with results reported in a comparative study of differentiation protocols for mouse ES cells (Shin et al., 2011). The efficiency of the short term protocol was proven by qPCR revealing the neural selection by increased Nestin expression and the onset of GAD1 and GAD2 expression under differentiation conditions. The increase of neuronal markers was coupled to the loss of pluripotency, demonstrated by the decrease of Oct 4 expression.

\section{Cell labeling efficiency}

The labeling of (embryonic) stem cells with paramagnetic contrast agents is not well established and only a few methodological reports exist. We tested several labeling techniques (pinocytosis, lipofection, calcium phosphate precipitation and electroporation), which were applied beforehand successfully to the upload of superparamagnetic contrast agents. Lipofection was reported to enable efficient labeling of ES cells and neural progenitor cells with the superparamagnetic contrast agent Sinerem (Kustermann et al., 2008). The single reports on lipofection of stem cells with paramagnetic contrast agents made use of Lipofectin and Lipofectamin (Rudelius et al., 2003), or Effectene (Shen et al., 2009). Recently, Guenoun et al. (2011) described a cationic Gd-DTPA liposome complex for enhanced labeling efficiency of mesenchymal stem cells. Our lipofection experiments revealed variable $\Delta \mathrm{R}_{1}$ from independent experiments in combination with a high viability and survival rate. The variability could be explained by the random lipofection process, in which the contrast agent-lipocomplex fuses with the cell membrane to form endosomes. Furthermore, relaxivity distortions are described for contrast agents entrapped in small cellular compartments like the endosomes (Strijkers et al., 2009; Terreno et al., 2006). The same endocytosis phenomenon occurs with the calcium phosphate precipitation (CaP) (de Duve et al., 1974), which was reported to yield highest labeling efficiency with Gd-DTPA for ES cells (Rudelius et al., 2003). CaP experiments reported here revealed no significant uptake compared to pinocytosis, although the protocol was adapted as described. Electroporation (EP) differs from these methods and allows entrapment of contrast agent into the cytosol. It was used for SPIO incorporation (Walczak et al., 2006) into neural stem cells and applied for rat hepatocarcinoma cells with Gd-HP-DO3A (Gadoteridol) (Terreno et al., 2006). EP of ES cells is the common molecular biology method for DNA transfer to the nucleus and yields - under these conditions - approximately 70\% cell survival (Siemen et al., 2005). The optimized protocol for contrast agent uptake described here resulted in $\geq 92 \%$ viability and $\geq 62 \%$ survival rate in the presence of $50 \mathrm{mM}$ Gd-DOTA. Only slightly lower viability $\geq 83 \%$ and survival rates $\geq 53 \%$ were obtained in the presence of $10 \mathrm{mM}$ Gd-DO3A-GAD. In conclusion, electroporation is the current method of choice for labeling stem cells with paramagnetic contrast agents provided that the protocol is adjusted for the specific cell type.

\section{In vitro and in vivo application}

The optimized electroporation protocol was applied to label ES cells and differentiated cells with Gd-DO3A-GAD. The cell pellets were subsequently measured at low field strength $(1 \mathrm{~T})$ and high field strength (7 T). Quantitative $\mathrm{T}_{1}$ maps confirmed higher relaxation rates at low field compared to high field strength, in agreement with expectation.

Differentiated cells showed a significant increase in $\mathrm{R} 1$ relative to undifferentiated cells indicating a GAD-specific activation of the Gdchelate in those cells. For a quantitative assessment of the contrast effect, the $\Delta R_{1}$ values should be normalized to the cell number, or even better, to be more precise, normalized to the $\mathrm{Gd}(\mathrm{III}) /$ protein content. Interestingly, the results of the contrast effect $\left(\Delta R_{1}\right)$ are found independent of the normalization procedure at low field. However, for high field, normalization of the labeling experiments to the $\mathrm{Gd}(\mathrm{III}) /$ protein content revealed lower $\Delta \mathrm{R}_{1}$ values than the normalization to cell numbers. This is explained by the variable uptake of the contrast agent in individual cells and the actual number of GABAergic neurons in the preparation. Despite this strongly field dependent contrast decrease at high field strength, these results underline the possibility of using the Gd-DO3A-GAD also at high-field MRI in combination with an accurate calculation of the actual contrast agent uptake.

Besides the variety of novel contrast agents responsive to cellular metabolites, nucleic acids, proteins or even metal ions, temperature and the $\mathrm{pH}$ (Yoo and Pagel, 2008), only few examples of a successful in vivo application have been reported. The $\beta-G$ al responsive contrast agent introduced by Moats et al. (1997) was used to visualize LacZ gene expression in living frog embryos, yielding a 57\% enhancement in the $\beta$-Gal positive regions (Louie et al., 2000). Furthermore, we reported in a previous implantation study the possibility to detect lipase expression. Dendritic cells labeled with a lipase responsive contrast agent were imaged after implantation into the rat brain (Himmelreich et al., 2006). The present study is the first proof-ofprinciple to discriminate between engrafted stem cells and GABAergic neurons in vivo using MRI. The change in $T_{1}$ relaxation rate was analyzed quantitatively for the in vitro and in vivo experiments, yielding $\geq 200 \mathrm{~ms}$ difference in $T_{1}$ in vitro and $\geq 100 \mathrm{~ms}$ in vivo. However, the normalization procedure cannot be applied to the in vivo condition directly (Himmelreich and Dresselaers, 2009), because of missing methods to determine the actual number of cells in the graft and the Gd(III) content precisely. This would be important for future experiments evaluating the relaxometric changes after onset of GAD expression on a quantitative basis.

\section{Conclusion}

Application of this cell-specific responsive MRI contrast agent permits in vivo monitoring of stem cell differentiation into GABAergic neurons, thus opening the door to follow cell fate of grafts in longitudinal noninvasive studies. Imaging of the inhibitory neuron specific GAD enzyme will offer invaluable diagnostic impact for cell replacement therapies for cerebral diseases such as stroke or Huntington's disease. With the here presented proof-of-principle imaging of the selective expression of GAD in differentiated ES cells, we provide a basis for future noninvasive molecular imaging approaches in stem cell research.

\section{Acknowledgment}

We gratefully acknowledge M. Diedenhofen, N. Henn, and M. Nelles for their excellent technical support. This work was funded in part by ENCITE EU-FP7 (HEALTH-F5-2008-201842), the Volkswagen Foundation (I/83 443), the region Piemonte (PIIMDMT and NanoIGT projects), the Italian Consortium CIRCMSB, grants of the EC-FP6 (MC-IRG-013080) and the KU Leuven (PF 10/017 IMIR).

\section{References}

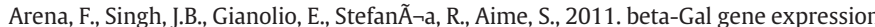
MRI reporter in melanoma tumor cells. Design, synthesis, and in vitro and in vivo testing of a Gd(III) Containing probe forming a high relaxivity, melanin-like structure upon $\beta$-gal enzymatic activation. Bioconjug. Chem.

Arvidsson, S. Kwasniewski, M. Riano-Pachon, D.M. Mueller-Roeber, B, 2008. QuantPrime - a flexible tool for reliable high-throughput primer design for quantitative PCR. BMC Bioinformatics 9 (1), 465.

Benes, F.M., Lim, B., Matzilevich, D., Walsh, J.P., Subburaju, S., Minns, M., 2007. Regulation of the GABA cell phenotype in hippocampus of schizophrenics and bipolars. Proc. Natl. Acad. Sci. U. S. A. 104, 10164-10169.

Brambilla, P., Perez, J., Barale, F., Schettini, G., Soares, J.C., 2003. GABAergic dysfunction in mood disorders. Mol. Psychiatry 8 (721-737), 715. 
Chen, C., Okayama, H., 1987. High-efficiency transformation of mammalian cells by plasmid DNA. Mol. Cell. Biol. 7, 2745-2752.

de Duve, C., de Barsy, T., Poole, B., Trouet, A., Tulkens, P., Van Hoof, F., 1974. Commentary. Lysosomotropic agents. Biochem. Pharmacol. 23, 2495-2531.

Deng, Y.P., Albin, R.L., Penney, J.B., Young, A.B., Anderson, K.D., Reiner, A., 2004. Differential loss of striatal projection systems in Huntington's disease: a quantitative immunohistochemical study. J. Chem. Neuroanat. 27, 143-164.

Di Cristo, G., 2007. Development of cortical GABAergic circuits and its implications for neurodevelopmental disorders. Clin. Genet. 72, 1-8.

Graham, F.L., van der Eb, A.J., 1973. A new technique for the assay of infectivity of human adenovirus 5 DNA. Virology 52, 456-467.

Guenoun, J., Koning, G.A., Doeswijk, G., Bosman, L., Wielopolski, P.A., Krestin, G.P., Bernsen, M.R., 2011. Cationic Gd-DTPA liposomes for highly efficient labeling of mesenchymal stem cells and cell tracking with MRI. Cell Transplant 21 (1), 191-205.

Himmelreich, U., Dresselaers, T., 2009. Cell labeling and tracking for experimenta models using magnetic resonance imaging. Methods 48 (2), 112-124.

Himmelreich, U., Aime, S., Hieronymus, T., Justicia, C., Uggeri, F., Zenke, M., Hoehn, M. 2006. A responsive MRI contrast agent to monitor functional cell status. Neuroimage 32, 1142-1149.

Hoehn, M., Himmelreich, U., 2006. In vivo molecular MR imaging - potentials and limits. In: Webb, G.A. (Ed.), Annual Reports in NMR Spectroscopy - Handbook of Modern Magnetic Resonance, Section Medical Science. Kluwer Academic Publisher, London, pp. 133-148.

Hoehn, M., Himmelreich, U., Kruttwig, K., Wiedermann, D., 2008. Molecular and cellular MR imaging: potentials and challenges for neurological applications. J. Magn. Reson. Imaging 27, 941-954.

Kruttwig, K., 2009. Molecular labelling strategies for cellular optical and magnetic resonance tomographic visualisation. Aachen, Techn. Hochsch., Diss.

Kustermann, E., Himmelreich, U., Kandal, K., Geelen, T., Ketkar, A., Wiedermann, D. Strecker, C., Esser, J., Arnhold, S., Hoehn, M., 2008. Efficient stem cell labeling for MRI studies. Contrast Media Mol. Imaging 3, 27-37.

Laemmli, U.K., 1970. Cleavage of structural proteins during the assembly of the head of bacteriophage T4. Nature 227, 680-685.

Lee, S.H., Lumelsky, N., Studer, L., Auerbach, J.M., McKay, R.D., 2000. Efficient generation of midbrain and hindbrain neurons from mouse embryonic stem cells. Nat. Biotechnol. 18, 675-679.

Louie, A.Y., Huber, M.M., Ahrens, E.T., Rothbacher, U., Moats, R., Jacobs, R.E., Fraser, S.E. Meade, T.J., 2000. In vivo visualization of gene expression using magnetic resonance imaging. Nat. Biotechnol. 18, 321-325.

Malizia, A.L., Cunningham, V.J., Bell, C.J., Liddle, P.F., Jones, T., Nutt, D.J., 1998. Decreased brain $\operatorname{GABA}(\mathrm{A})$-benzodiazepine receptor binding in panic disorder: preliminary results from a quantitative PET study. Arch. Gen. Psychiatry 55, 715-720.

Moats, R.A., Fraser, S.E., Meade, T.J., 1997. Ein “intelligentes” Reagens für die NMRBildgebung zur Bestimmung spezifischer enzymatischer Aktivität. Angew. Chem. Int. Ed. Engl. 36, 726 .

Modo, M., Hoehn, M., Bulte, J.W., 2005. Cellular MR imaging. Mol. Imaging 4, 143-164.

Mueller, P.B., Langemann, H., 1962. Distribution of glutamic acid decarboxylase activity in human brain. J. Neurochem. 9, 399-401.
Napolitano, R., Pariani, G., Fedeli, F., Aswendt, M., Aime, S., Gianolio, E., in press. Synthesis and Relaxometric Characterization of a MRI Gd-based probe responsive to GAD enzymatic activity. Bioconjugate Chem.

Neumann, E., Schaefer-Ridder, M., Wang, Y., Hofschneider, P.H., 1982. Gene transfer into mouse lyoma cells by electroporation in high electric fields. EMBO J. 1, 841-845.

Okabe, S., Forsberg-Nilsson, K., Spiro, A.C., Segal, M., McKay, R.D., 1996. Development of neuronal precursor cells and functional postmitotic neurons from embryonic stem cells in vitro. Mech. Dev. 59, 89-102.

Paul, S.M., 1995. Psychopharmacology: the fourth generation of progress. In: Bloom, F.E., Kupfer, D.J. (Eds.), Drug Development Research, 35, pp. 185-188.

Pfaffl, M.W., Horgan, G.W., Dempfle, L., 2002. Relative expression software tool (REST) for group-wise comparison and statistical analysis of relative expression results in real-time PCR. Nucleic Acids Res. 30, e36.

Reiner, A., Albin, R.L., Anderson, K.D., D'Amato, C.J., Penney, J.B., Young, A.B., 1988. Differential loss of striatal projection neurons in Huntington disease. Proc. Natl. Acad. Sci. U. S. A. $85,5733-5737$.

Roberts, E., Simonsen, D.G., 1963. Some properties of L-glutamic decarboxylase in mouse brain. Biochem. Pharmacol. 12, 113-134.

Rudelius, M., Daldrup-Link, H.E., Heinzmann, U., Piontek, G., Settles, M., Link, T.M., Schlegel, J., 2003. Highly efficient paramagnetic labelling of embryonic and neuronal stem cells. Eur. J. Nucl. Med. Mol. Imaging 30, 1038-1044.

Saji, M., Cohen, M., Blau, A.D., Wessel, T.C., Volpe, B.T., 1994. Transient forebrain ischemia induces delayed injury in the substantia nigra reticulata: degeneration of GABA neurons, compensatory expression of GAD mRNA. Brain Res. 643, 234-244.

Schwartz-Bloom, R.D., Sah, R., 2001. Gamma-aminobutyric acid(A) neurotransmission and cerebral ischemia. J. Neurochem. 77, 353-371.

Shen, J., Cheng, L.N., Zhong, X.M., Duan, X.H., Guo, R.M., Hong, G.B., 2009. Efficient in vitro labeling rabbit neural stem cell with paramagnetic Gd-DTPA and fluorescent substance. Eur. J. Radiol. 75, 397-405.

Shin, E., Palmer, M.J., Li, M., Fricker, R.A., 2011. GABAergic neurons from mouse embryonic stem cells possess functional properties of striatal neurons in vitro, and develop into striatal neurons in vivo in a mouse model of Huntington's disease. Stem Cell Rev. 767 (4), Pages: 333-341.

Siemen, H., Nix, M., Endl, E., Koch, P., Itskovitz-Eldor, J., Brustle, O., 2005. Nucleofection of human embryonic stem cells. Stem Cells Dev. 14, 378-383.

Strijkers, G. Hak, S, Kok, M. B. Springer Jr. CS, Nicolay, K, 2009. Three-compartment $\mathrm{T}(1)$ relaxation model for intracellular paramagnetic contrast agents. Magn. Reson. Med. 61, 1049-1058

Terreno, E., Geninatti Crich, S., Belfiore, S., Biancone, L., Cabella, C., Esposito, G., Manazza, A.D., Aime, S., 2006. Effect of the intracellular localization of a Gdbased imaging probe on the relaxation enhancement of water protons. Magn. Reson. Med. 55, 491-497.

Walczak, P. Ruiz-Cabello, J., Kedziorek, D.A, Gilad, A.A, Lin, S., Barnett, B, Qin, L. Levitsky, H., Bulte, J.W., 2006. Magnetoelectroporation: improved labeling of neural stem cells and leukocytes for cellular magnetic resonance imaging using a single FDA-approved agent. Nanomedicine 2, 89-94.

Willems, E., Mateizel, I., Kemp, C., Cauffman, G., Sermon, K., Leyns, L., 2006. Selection of reference genes in mouse embryos and in differentiating human and mouse ES cells. Int. J. Dev. Biol. 50, 627-635.

Yoo, B., Pagel, M.D., 2008. An overview of responsive MRI contrast agents for molecular imaging. Front. Biosci. 13, 1733-1752. 